\title{
Detecting Large-Scale System Problems by Mining Console Logs
}

\author{
Wei $\mathrm{Xu}^{*} \quad$ Ling Huang ${ }^{\dagger} \quad$ Armando Fox* ${ }^{*}$ David Patterson* Michael I. Jordan* \\ ${ }^{*}$ EECS Department $\quad{ }^{\dagger}$ Intel Labs Berkeley \\ University of California at Berkeley, USA Berkeley, CA, USA \\ \{xuw,fox,pattrsn,jordan\}@cs.berkeley.edu ling.huang@intel.com
}

\begin{abstract}
Surprisingly, console logs rarely help operators detect problems in large-scale datacenter services, for they often consist of the voluminous intermixing of messages from many software components written by independent developers. We propose a general methodology to mine this rich source of information to automatically detect system runtime problems. We first parse console logs by combining source code analysis with information retrieval to create composite features. We then analyze these features using machine learning to detect operational problems. We show that our method enables analyses that are impossible with previous methods because of its superior ability to create sophisticated features. We also show how to distill the results of our analysis to an operator-friendly one-page decision tree showing the critical messages associated with the detected problems. We validate our approach using the Darkstar online game server and the Hadoop File System, where we detect numerous real problems with high accuracy and few false positives. In the Hadoop case, we are able to analyze 24 million lines of console logs in 3 minutes. Our methodology works on textual console logs of any size and requires no changes to the service software, no human input, and no knowledge of the software's internals.
\end{abstract}

\section{Introduction}

When a datacenter-scale service consisting of hundreds of software components running on thousands of computers misbehaves, developer-operators need every tool at their disposal to troubleshoot and diagnose operational problems. Ironically, there is one source of information that is built into almost every piece of software that provides detailed information that reflects the original developers' ideas about noteworthy or unusual events, but is typically ignored: the humble console log.

Since the dawn of programming, developers have used everything from printf to complex logging and monitoring libraries $[8,9]$ to record program variable values, trace execution, report runtime statistics, and even print out full-sentence messages designed to be read by a human - usually by the developer herself. However, modern large-scale services usually combine large opensource components authored by hundreds of developers, and the people scouring the logs-part integrator, part developer, part operator, and charged with fixing the problem-are usually not the people who chose what to log or why. (We'll use the term operator to represent a potentially diverse set of people trying to detect operational problems.) Furthermore, even in well-tested code, many operational problems are dependent on the deployment and runtime environment and cannot be easily reproduced by the developer. To make things worse, modern systems integrate external (often open source) components that are frequently revised or upgraded, which may change what's in the logs or the relevance of certain messages. Keeping up with this churn rate exacerbates the operators' dilemma. Our goal is to provide them with better tools to extract value from the console logs.

As logs are too large to examine manually $[14,22]$ and too unstructured to analyze automatically, operators typically create $a d$ hoc scripts to search for keywords such as "error" or "critical," but this has been shown to be insufficient for determining problems [14, 22]. Rule-based processing [24] is an improvement, but the operators' lack of detailed knowledge about specific components and their interactions makes it difficult to write rules that pick out the most relevant sets of events for problem detection. Instead of asking users to search, we provide tools to automatically find "interesting" log messages.

Since unusual log messages often indicate the source of the problem, it is natural to formalize log analysis as an anomaly detection problem in machine learning. However, it is not always the case that the presence, absence or frequency of a single type of message is sufficient to pinpoint the problem; more often, a problem manifests as an abnormality in the relationships among different types of log messages (correlations, relative frequencies, and so on). Therefore, instead of analyzing the words in textual logs (as done, for example, in [27]), we create features that accurately capture various correlations among $\log$ messages, and perform anomaly detection on these features. Creating these features requires augmenting log parsing with information about source code; our 
method for doing this augmentation is part of our contribution.

We studied logs and source code of many popular software systems used in Internet services, and observed that a typical console log is much more structured than it appears: the definition of its "schema" is implicit in the log printing statements, which can be recovered from program source code. This observation is key to our log parsing approach, which yields detailed and accurate features. Given the ubiquitous presence of open-source software in many Internet systems, we believe the need for source code is not a practical drawback to our approach.

Our contribution is a general four-step methodology that allows machine learning and information retrieval techniques to be applied to free-text $\operatorname{logs}$ to find the "needles in the haystack" that might indicate operational problems, without any manual input. Specifically, our methodology involves the following four contributions:

1) A technique for analyzing source code to recover the structure inherent in console logs;

2) The identification of common information in logsstate variables and object identifiers - and the automatic creation of features from the logs (exploiting the structure found) that can be subjected to analysis by a variety of machine learning algorithms;

3) Demonstration of a machine learning and information retrieval methodology that effectively detects unusual patterns or anomalies across large collections of such features extracted from a console log;

4) Where appropriate, automatic construction of a visualization that distills the results of anomaly detection in a compact and operator-friendly format that assumes no understanding of the details of the algorithms used to analyze the features.

The combination of elements in our approach, including our novel combination of source code analysis with log analysis and automatic creation of features for anomaly detection, enables a level of detail in log analysis that was previously impossible due to the inability of previous methods to correctly identify the features necessary for problem identification.

Our approach requires no changes to existing software and works on existing textual console logs of any size, and some of the more computationally expensive steps are embarrassingly parallel, allowing us to run them as Hadoop [2] map-reduce jobs using cloud computing, achieving nearly linear speedup for a few dollars per run.

We evaluate our approach and demonstrate its capability and scalability with two real-world systems: the Darkstar online game server [28] and the Hadoop File System. For Darkstar, our method accurately detects performance anomalies immediately after they happen and provides hints as to the root cause. For Hadoop, we detect runtime anomalies that are commonly overlooked, starting: xact 325 is COMMITTING

starting: xact 346 is ABORTING

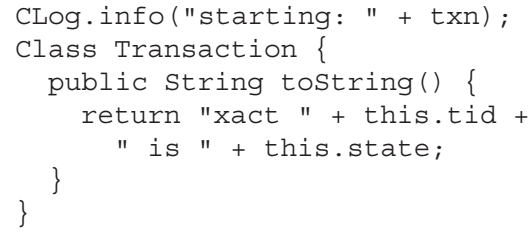

Figure 1: Top: two lines from a simple console log. Bottom: Java code that could produce these lines.

and distill over 24 million lines of console logs (collected from 203 Hadoop nodes) to a one-page decision tree that a domain expert can readily understand. This automated process can be done with Hadoop map-reduce on 60 Amazon EC2 nodes within 3 minutes.

Section 2 provides an overview of our approach, Section 3 describes our log parsing technique in detail, Sections 4 and 5 present our solutions for feature creation and anomaly detection, Section 6 evaluates our approach and discusses the visualization technique, Section 7 discusses extensions and provide suggestions to improve $\log$ quality, Section 8 summarizes related work, and Section 9 draws some conclusions.

\section{Overview of Approach}

\subsection{Information buried in textual $\operatorname{logs}$}

Important information is buried in the millions of lines of free-text console logs. To analyze logs automatically, we need to create high quality features, the numerical representation of $\log$ information that is understandable by a machine learning algorithm. The following three key observations lead to our solution to this problem.

Source code is the "schema" of logs. Although console logs appear in free text form, they are in fact quite structured because they are generated entirely from a relatively small set of log printing statements in the system.

Consider the simple console log excerpt and the source code that generated it in Figure 1. Intuitively, it is easier to recover the log's hidden "schema" using the source code information (especially for a machine). Our method leverages source code analysis to recover the inherit structure of logs. The most significant advantage

\begin{tabular}{|l|l|l|}
\hline Variable & Examples & $\begin{array}{l}\text { Distinct } \\
\text { values }\end{array}$ \\
\hline Identifiers & $\begin{array}{l}\text { transaction_id in Darkstar; block_id } \\
\text { in Hadoop file system; cache_key in the } \\
\text { Apache web server; task_id in Hadoop } \\
\text { map reduce. }\end{array}$ & many \\
\hline $\begin{array}{l}\text { State } \\
\text { Vars }\end{array}$ & $\begin{array}{l}\text { Transaction execution state in Darkstar; } \\
\text { Server name for each block in Hadoop file }\end{array}$ & \\
& $\begin{array}{l}\text { POStem; HTTP status code (200, 404); } \\
\text { POSIX process return values; }\end{array}$ & \\
\hline
\end{tabular}

Table 1: State variables and identifiers 


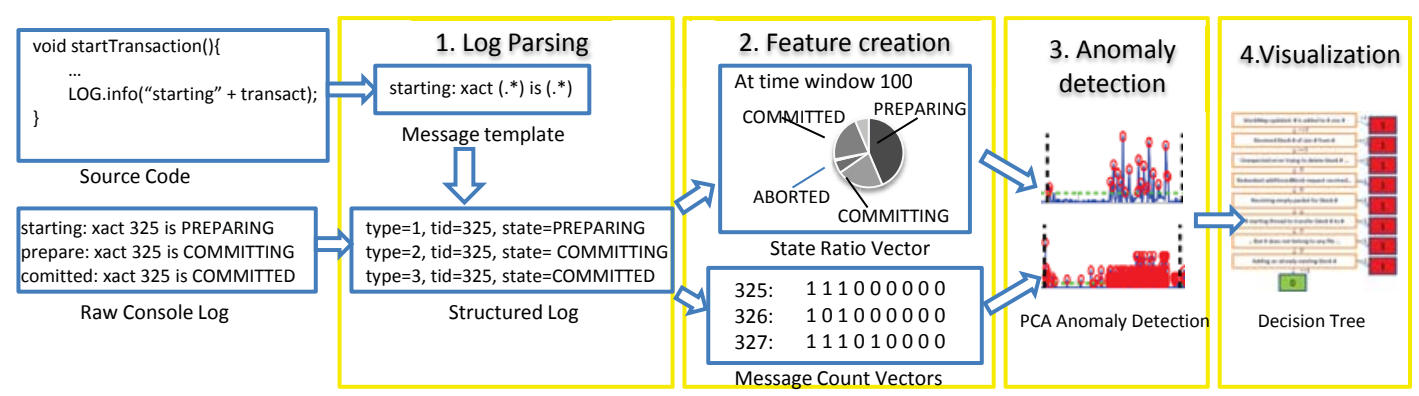

Figure 2: Overview of console log analysis work flow.

of our approach is that we are able to accurately parse all possible log messages, even the ones rarely seen in actual logs. In addition, we are able to eliminate most heuristics and guesses for log parsing used by existing solutions.

Common log structures lead to useful features. A person usually reads the log messages in Figure 1 as a constant part (starting: xact ... is) and multiple variable parts (325/326 and COMMITTING/ABORTING). In this paper, we call the constant part the message type and the variable part the message variable.

Message types-marked by constant strings in a log message - are essential for analyzing console logs and have been widely used in earlier work [17]. In our analysis, we use the constant strings solely as markers for the message types, completely ignoring their semantics as English words, which is known to be ambiguous [22].

Message variables carry crucial information as well. In contrast to prior work that focuses on numerical variables [17, 22, 35], we identified two important types of message variables for problem detection by studying logs from many systems and by interviewing Internet service developers/operators who heavily use console logs. We acknowledge that $\log$ s also contain other types of message variables such as timestamps and various counts. We do not discuss those variables in this paper as they have been well studied in existing work [27].

Identifiers are variables used to identify an object manipulated by the program (e.g., the transaction ids 325 and 346 in Figure 1), while state variables are labels that enumerate a set of possible states an object could have in program (e.g. COMMITTING and ABORTING in Figure 1). Table 1 provides extra examples of such variables. We can determine whether a given variable is an identifier or a state variable progmatically based on its frequency in console logs. Intuitively, state variables have a small number of distinct values while identifiers take a large number of distinct values (detailed in Section 4).

Message types and variables contain important runtime information useful to the operators. However, lacking tools to extract these structures, operators either ignore them, or spend hours greping and manually examining log messages, which is tedious and inefficient.

Our accurate log parsing allows us to use structured information such as message types and variables to automatically create features that capture information conveyed in logs. To our knowledge, this is the first work extracting information at this fine level of granularity from console logs for problem detection.

Messages are strongly correlated. When log messages are grouped properly, there is a strong and stable correlation among messages within the same group. For example, messages containing a certain file name are likely to be highly correlated because they are likely to come from logically related execution steps in the program.

A group of related messages is often a better indicator of problems than individual messages. Many anomalies are only indicated by incomplete message sequences. For example, if a write operation to a file fails silently (perhaps because the developers do not handle the error correctly), no single error message is likely to indicate the failure. By correlating messages about the same file, however, we can detect such cases by observing that the expected "closing file" message is missing. Previous work grouped logs by time windows only, and the detection accuracy suffers from noise in the correlation $[14,27,35]$. In contrast, we create message groups based on more accurate information, such as the message variables described above. In this way, the correlation is much stronger and more readily encoded so that the abnormal correlations also become easier to detect.

\subsection{Workflow of our approach}

Figure 2 shows the four steps in our general framework for mining console logs.

1) Log parsing. We first convert a log message from unstructured text to a data structure that shows the message type and a list of message variables in (name, value) pairs. We get all possible log message template strings from the source code and match these templates to each log message to recover its structure (that is, message type and variables). Our experiments show that we can achieve high parsing accuracy in real-world systems.

There are systems that use structured tracing only, such as BerkeleyDB (Java edition). In this case, because logs are already structured, we can skip this first step and directly apply our feature creation and anomaly detec- 


\begin{tabular}{|c|c|c|c|c|c|c|c|c|c|}
\hline System & Lang & Logger & Msg Construction & LOC & LOL & Vars & Parse & ID & ST \\
\hline \multicolumn{10}{|l|}{ Operating system } \\
\hline Linux (Ubuntu) & $\mathrm{C}$ & custom & printk + printf wrap & $7477 \mathrm{k}$ & 70817 & 70506 & $\mathrm{Y}$ & $\mathrm{Y}^{\mathrm{b}}$ & $\mathrm{Y}$ \\
\hline \multicolumn{10}{|c|}{ Low level Linux services } \\
\hline Bootp & $\mathrm{C}$ & custom & printf wrap & $11 \mathrm{k}$ & 322 & 220 & $\bar{Y}$ & $\mathrm{~N}$ & $\mathrm{~N}$ \\
\hline DHCP server & $\mathrm{C}$ & custom & printf wrap & $23 \mathrm{k}$ & 540 & 491 & $\bar{Y}$ & $\mathrm{Y}^{b}$ & $\bar{Y}$ \\
\hline DHCP client & $\mathrm{C}$ & custom & printf wrap & $5 \mathrm{k}$ & 239 & 205 & $\mathrm{Y}$ & $\mathrm{Y}^{b}$ & $\mathrm{Y}$ \\
\hline ftpd & $\mathrm{C}$ & custom & printf wrap & $3 \mathrm{k}$ & 66 & 67 & $\mathrm{Y}$ & $\mathrm{Y}$ & $\mathrm{N}$ \\
\hline openssh & $\bar{C}$ & custom & printf wrap & $124 \mathrm{k}$ & 3341 & 3290 & $\bar{Y}$ & $\bar{Y}$ & $\bar{Y}$ \\
\hline crond & $\mathrm{C}$ & printf & printf wrap & $7 \mathrm{k}$ & 112 & 131 & $\mathrm{Y}$ & $\mathrm{N}$ & $\mathrm{Y}$ \\
\hline Kerboros 5 & $\mathrm{C}$ & custom & printf wrap & $44 \mathrm{k}$ & 6261 & 4971 & $\mathrm{Y}$ & $\mathrm{Y}$ & $\mathrm{Y}$ \\
\hline iptables & $\mathrm{C}$ & custom & printf wrap & $52 \mathrm{k}$ & 2341 & 1528 & $\mathrm{Y}$ & $\mathrm{N}$ & $\bar{Y}$ \\
\hline Samba 3 & $\mathrm{C}$ & custom & printf wrap & $566 \mathrm{k}$ & 8461 & 6843 & $\mathrm{Y}$ & $\mathrm{Y}$ & $\mathrm{Y}$ \\
\hline \multicolumn{10}{|c|}{ Internet service building blocks } \\
\hline Apache2 & $\mathrm{C}$ & custom & printf wrap & $312 \mathrm{k}$ & 4008 & 2835 & $\mathrm{Y}$ & $\bar{Y}$ & $\mathrm{Y}$ \\
\hline mysql & $\mathrm{C}$ & custom & printf wrap & $714 \mathrm{k}$ & 5092 & 5656 & $\mathrm{Y}$ & $\mathrm{Y}^{\mathrm{b}}$ & $\mathrm{Y}^{\mathrm{b}}$ \\
\hline postgresql & $\mathrm{C}$ & custom & printf wrap & $740 \mathrm{k}$ & 12389 & 7135 & $\mathrm{Y}$ & $\mathrm{Y}^{\mathrm{b}}$ & $\mathrm{Y}^{\mathrm{b}}$ \\
\hline Squid & $\mathrm{C}$ & custom & printf wrap & $148 \mathrm{k}$ & 2675 & 2740 & $\bar{Y}$ & $\mathrm{Y}$ & $\bar{Y}$ \\
\hline Jetty & Java & $\log 4 j$ & string concatenation & $138 \mathrm{k}$ & 699 & 667 & $\mathrm{Y}$ & $\mathrm{Y}$ & $\mathrm{Y}$ \\
\hline Lucene & Java & custom & custom log function & $217 \mathrm{k}$ & 143 & 159 & $\mathrm{Y}^{\mathrm{a}}$ & $\mathrm{Y}$ & $\mathrm{N}$ \\
\hline BDB (Java) & Java & custom & custom structured trace & $260 \mathrm{k}$ & - & - & - & $\bar{Y}$ & $\bar{N}$ \\
\hline \multicolumn{10}{|c|}{ Distributed systems } \\
\hline Hadoop & Java & custom $\log 4 \mathrm{j}$ & string concatenation & $173 \mathrm{k}$ & 911 & 1300 & $\mathrm{Y}$ & $\mathrm{Y}$ & $\mathrm{Y}$ \\
\hline Darkstar & Java & jdk-log & Java format string & $90 \mathrm{k}$ & 578 & 658 & $\bar{Y}$ & $\mathrm{Y}^{b}$ & $\mathrm{Y}^{\mathrm{b}}$ \\
\hline Nutch & Java & $\log 4 j$ & string concatenation & $64 \mathrm{k}$ & 507 & 504 & $\mathrm{Y}$ & $\mathrm{Y}$ & $\mathrm{Y}$ \\
\hline Cassandra & Java & $\log 4 j$ & string concatenation & $46 \mathrm{k}$ & 393 & 437 & $\bar{Y}$ & $\mathrm{~N}$ & $\bar{Y}$ \\
\hline Storage Prototype & $\mathrm{C}$ & custom & custom structured trace & c & $-^{\mathrm{c}}$ & $-^{\mathrm{c}}$ & $-^{c}$ & $\mathrm{Y}$ & $\overline{\mathrm{Y}}$ \\
\hline
\end{tabular}

${ }^{a}$ Logger class is not consistent in every module, so we need to manually specify the logger function name for each module.

${ }^{b}$ System prints minimal amount of logs by default, so we need to enable debug logging.

${ }^{c}$ Source code not available, but logs are well structured so manual parsing is easy.

Table 2: Console logging in popular software systems. LOC $=$ lines of codes in the system. LOL $=$ number of log printing statements. Vars $=$ number of variables reported in log messages. Parse $=$ whether our source analysis based parsing applies. ID $=$ whether identifier variables are reported. ST $=$ whether state variables are reported.

tion methods. Note that these structured logs still contain both identifiers and state variables. ${ }^{1}$

2) Feature creation. Next, we construct feature vectors from the extracted information by choosing appropriate variables and grouping related messages. In this paper, we focus on constructing the state ratio vector and the message count vector features, which are unexploited in prior work. In our experiments with two large-scale realworld systems, both features yield good detection results.

3) Anomaly detection. Then, we apply anomaly detection methods to mine feature vectors, labeling each feature vector as normal or abnormal. We find that the Principal Component Analysis (PCA)-based anomaly detection method [5] works very well with both features. This method is an unsupervised learning algorithm, in which all parameters can be either chosen automatically or tuned easily, eliminating the need for prior input from the operators. Although we use this specific machine learning algorithm for our case studies, it is not intrinsic

\footnotetext{
${ }^{1}$ In fact, the last system in Table 2 (Storage Prototype) is an anonymous research prototype with built-in customized structured traces. Without any context, even without knowing the functionality of the system, our feature creation and anomaly detection algorithm successfully discovered log segments that the developer found insightful.
}

to our approach, and different algorithms utilizing different extracted features could be readily "plugged in" to our framework.

4) Visualization. Finally, in order to let system integrators and operators better understand the PCA anomaly detection results, we visualize results in a decision tree [34]. Compared to the PCA-based detector, the decision tree provides a more detailed explanation of how the problems are detected, in a form that resembles the event processing rules [10] with which system integrators and operators are familiar.

\subsection{Case study and data collection}

We studied source code and $\log$ from 22 widely deployed open source systems. Table 2 summarizes the results. Although these systems are distinct in nature, developed in different languages by different developers at different times, 20 of the 22 systems use free text logs, and our source-code-analysis based log parsing applies to all of the 20 . Interestingly, we found that about $1 \%-5 \%$ of code lines are logging calls in most of the systems, but most of these calls are rarely, if ever, executed because they represent erroneous execution paths. It is almost impossible to maintain log-parsing rules manually 


\begin{tabular}{|r|r|r|r|}
\hline System & Nodes & Messages & Log Size \\
\hline Darkstar & 1 & $1,640,985$ & $266 \mathrm{MB}$ \\
\hline Hadoop (HDFS) & 203 & $24,396,061$ & $2412 \mathrm{MB}$ \\
\hline
\end{tabular}

Table 3: Data sets used in evaluation. Nodes=Number of nodes in the experiments.

with such a large number of distinct logger calls, which highlights our advantage of discovering message types automatically from source code. On average, a message reports a single variable. However, there are many messages, such as starting server that reports no variables, while other messages can report 10 or more.

Most C programs use printf style format strings for logging, although a large portion uses wrapper functions to generate standard information such as time stamps and severity levels. These wrappers, even if customized, can be detected automatically from the format string parameter. In contrast, Java programs usually use string concatenation to generate log messages and often rely on standard logger packages (such as $\log 4 j$ ). Analyzing these logging calls requires understanding data types, which we detail in Section 3. Our source-code-analysis based log parsing approach successfully works on most of them, and can find at least one of state variables and identifiers in 21 of the 22 systems in Table 2 (16 have both), confirming our assumption of their prevalence.

To be succinct yet reveal important issues in console log mining, we focus further discussion on two representative systems shown in Table 2: the Darkstar online game server and the Hadoop File System (HDFS). Both systems handle persistence, an important yet complicated function in large-scale Internet services. However, these two systems are different in nature. Darkstar focuses on small, time sensitive transactions, while HDFS is a file system designed for storing large files and batch processing. They represent two major open source contributors (Sun and Apache, respectively) with different coding and logging styles.

We collected logs from systems running on Amazon's Elastic Compute Cloud (EC2) and we also used EC2 to analyze these logs. Table 3 summarizes the log data sets we used. The Darkstar example revealed a behavior that strongly depended on the deployment environment, which led to problems when migrating from traditional server farms to clouds. In particular, we found that Darkstar did not gracefully handle performance variations that are common in the cloud-computing environment. By analyzing console logs, we found the reason for this problem, as discussed in detail in Section 6.2.

Satisfied with Darkstar results, to further evaluate our method we analyzed HDFS logs, which are much more complex. We collected HDFS logs from a Hadoop cluster running on over $200 \mathrm{EC} 2$ nodes, yielding 24 million lines of logs. We successfully extracted log segments in- dicating run-time performance problems that have been confirmed by Hadoop developers.

All log data are collected from unmodified off-theshelf systems. Console logs are written directly to local disks on each node and collected offline by simply copying $\log$ files, which shows the convenience (no instrumentation or configuration) of our log mining approach. In the HDFS experiment, we used the default logging level, while in the Darkstar experiment, we turned on debug logging (FINER level in the logging framework).

\section{Log Parsing with Source Code}

In addition to standard "fields" in console logs, such as timestamps, we focus on the free text part of a $\log$ message. For the log excerpt at the top of Figure 1, human readers would reasonably conclude that 325 , 346, COMMITTING and ABORTING are message variables while the rest are constant strings marking message types. They could then write a regular expression such as starting: xact $(. *)$ is $(. *)$ to "templatize" such $\log$ messages. We want to automate the process.

The difficulty. Unless the log itself is marked with formatting to distinguish these elements, we must "templatize" automatically. As discussed in Section 2.1, it is much easier for a machine to use the source code as the "schema" for console logs. If the software is written in a language like $\mathrm{C}$, it is likely that the template can be directly inferred from printf variants that generate the messages, such as fprintf(LOG, "starting: xact $\% d$ is $\% s ")$, with the various escapes ( $\% \mathrm{~d}$, $\% \mathrm{f}$, and so on) telling us something about the types of the variables. However, it is more challenging in object-oriented (OO) languages such as Java, which are increasingly used for open source software (Table 2). Consider the excerpt of Java source shown in the bottom half of Figure 1, which generated the two example log lines. Clearly, the tid variable of the txn object corresponds to the identifiers 325 and 346 in the log message of Figure 1, and the state variable corresponds to the labels COMMITTING and ABORTING. Trying to extract a regular expression by simply "grepping" the source code would only give us starting: $(. *)$ (line 1), which does not distinguish tid and state as separate features with distinct ranges of possible values. Critically, as we will show later, we need this finer level of feature resolution to find "interesting" problems.

Three reasons cause this difficulty to arise in OO languages. First, we need to know that CLog identifies a logger object; that is, knowing the name of the logger class is not enough. Second, the $\mathrm{OO}$ idiom for printing is for an object to implement a tostring () method that returns a printable representation of itself for interpolation into a string; in this example, the tostring () method of the abstract type Transaction actually reveals the 


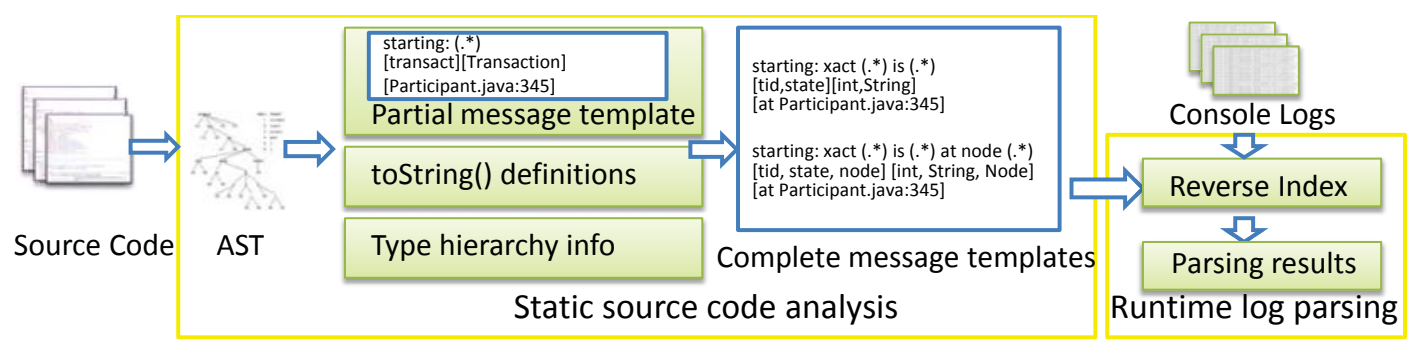

Figure 3: Using source code information to parse console logs.

underlying structure of the log message. Third, due to class inheritance, the actual tostring () method used in a particular call might be defined in a subclass rather than the base class of the logger object.

Our parsing approach. All three reasons are addressed by our log parsing method that consists of two steps: a static source code analysis step and the runtime log parsing step, as Figure 3 illustrates. In particular, we do not claim to handle every situation correctly (despite extensive support for language idioms), but we do show that some of the important features used in our results cannot be extracted using existing log parsing techniques.

The static source code analysis step takes program source (and possibly the names of the logger class) as input. In this step, we first generate the source code's abstract syntax tree (AST) [1], a popular data structure for traversing and analyzing source code. We use the AST implementations built into the open-source Eclipse IDE [25]. We use the AST to identify all method calls on objects of the classes (or their subclasses), recording the filename and line number of the call. Each call gives us only a partial message template, since the template may involve interpolation of objects of nonprimitive types, as in line 1 of the source code excerpt in Figure 1. We then enumerate all tostring ( ) calls in all classes, and look at the string formatting statements in those calls to deduce the types of variables in message templates, substituting this type information back into the partial templates. We do this recursively until all templates interpolate only primitive types; if no tostring () method can be found for a particular variable anywhere along its inheritance path, we assume that that variable can take on any string value and we do no further semantic interpretation. A single pass can accomplish all of these operations over the AST. The output of the process is the complete message templates, with a data structure containing each message's template (regular expression), position in the source code, and the names and data types of all variables appearing in the message. We describe the details of the template extraction approach in Appendix A.

To parse the logs, we first compile all message templates into an Apache Lucene [11] reverse index [20], which allows us to quickly associate any log message with the corresponding template. Following established heuristics in log analysis [17, 30], we construct an index query from each log message by removing all numbers and special symbols. From the list of relevance-ranked candidate results returned by the reverse-index search, we pick the highest-ranked result that allows a regular expression match to succeed against the log message. We note that once the reverse index is constructed (it usually fits in memory), the parsing step is embarrassingly parallel; we implement it as a Hadoop map-reduce job by replicating the index to every worker node and partitioning the log among the workers, achieving near linear speedup. The map stage performs the reverse-index search; the reduce stage processing depends on the features to be constructed, and Section 4 shows 2 examples.

To summarize, unlike existing log-parsing methods, the fine granularity of structure revealed by our method enables analyses that are traditionally possible only with structured logs. Section 7 discusses the many intrinsic subtleties in source code analysis and log parsing.

\section{Feature Creation}

This section describes our technique for constructing features from parsed logs. We focus on two features, the state ratio vector and the message count vector, based on state variables and identifiers (see Section 2.1), respectively. The state ratio vector is able to capture the aggregated behavior of the system over a time window. The message count vector helps detect problems related to individual operations. Both features describe message groups constructed to have strong correlations among their members. The features faithfully capture these correlations, which are often good indicators of runtime problems. Although these features are from the same log, and similar in structure, they are constructed independently, and have different semantics.

\subsection{State variables and state ratio vectors}

State variables can appear in a large portion of log messages. In fact, $32 \%$ of the log messages from Hadoop and $28 \%$ of messages from Darkstar contain state variables.

In many systems, during normal execution the relative frequency of each value of a state variable in a time window usually stays the same. For example, in Darkstar, the ratio between ABORTING and COMMITTING is very stable during normal execution, but changes significantly when 
a problem occurs. Notice that the actual number does not matter (as it depends on workload), but the ratio among different values matters.

We construct state ratio vectors $\mathbf{y}$ to encode this correlation: Each state ratio vector represents a group of state variables in a time window, while each dimension of the vector corresponds to a distinct state variable value, and the value of the dimension is how many times this state value appears in the time window.

In creating features based on state variables we used an automatic procedure that combined two desiderata: 1) message variables should be frequently reported, but 2) they should range across a small constant number of distinct values that do not depend on the number of messages. Specifically in our experiments, we chose state variables that were reported at least $0.2 \mathrm{~N}$ times, with $N$ the number of messages, and had a number of distinct values not increasing with $N$ for large values of $N$ (e.g., more than a few thousand). Our results were not sensitive to the choice of 0.2 .

The time window size is also automatically determined. Currently we choose a size that allows the variable to appear at least $10 D$ times in $80 \%$ of all the time windows, where $D$ is the number of distinct values. This choice of time window allows the variable to appear enough times in each window to make the count statistically significant [4] while keeping the time window small enough to capture transient problems. We tried with other parameters than 10 and $80 \%$ and we did not see a significant change in detection results.

We stack all $n$-dimensional y's from $m$ time windows to construct the $m \times n$ state ratio matrix $\mathbf{Y}^{s}$.

\subsection{Identifiers and message count vectors}

Identifiers are also prevalent in logs. For example, almost $50 \%$ of messages in HDFS logs contain identifiers. We observe that all log messages reporting the same identifier convey a single piece of information about the identifier. For instance, in HDFS, there are multiple log messages about a block when the block is allocated, written, replicated, or deleted. By grouping these messages, we get the message count vector, which is similar to an execution path [8] (from custom instrumentation).

To form the message count vector, we first automatically discover identifiers, then group together messages with the same identifier values, and create a vector per group. Each vector dimension corresponds to a different message type, and the value of the dimension tells how many messages of that type appear in the message group.

The structure of this feature is analogous to the bag of words model in information retrieval [6]. In our application, the "document" is the message group. The dimensions of the vector consist of the union of all useful message types across all groups (analogous to all possible "terms"), and the value of a dimension is the number

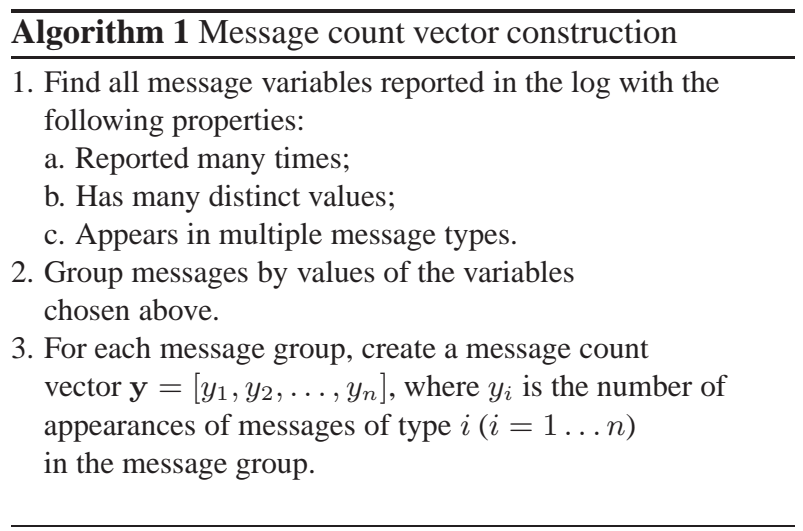

of appearances of the corresponding message types in a group (corresponding to "term frequency").

Algorithm 1 summarizes our three-step process for feature construction. We now try to provide intuition behind the design choices in this algorithm.

In the first step of the algorithm, we automatically choose identifiers (we do not want to require operators to specify a search key). The intuition is that if a variable meets the three criteria in step 1 of Algorithm 1, it is likely to identify object such as transactions. The frequency/distinct value pattern of identifiers is very different from other variables, so it is easy to discover identifiers ${ }^{2}$. We have very few false selections in all data sets, and the small number of false choices is easy to eliminate by a manual examination.

In the second step, the message group essentially describes an execution path, with two major differences. First, not every processing step is necessarily represented in the console logs. Since the logging points are hand chosen by developers, it is reasonable to assume that logged steps should be important for diagnosis. Second, correct ordering of messages is not guaranteed across multiple nodes, due to unsynchronized clocks across many computers. This ordering might be a problem for diagnosing synchronization-related problems, but it is still useful in identifying many kinds of anomalies.

In the third step, we use the bag of words model [6] to represent the message group because: 1) it does not require ordering among terms (message types), and 2) documents with unusual terms are given more weight in document ranking. In our case, the rare log messages are indeed likely to be more important.

We gather all the message count vectors to construct message count matrix $\mathbf{Y}^{m}$ as an $m \times n$ matrix where each row is a message count vector $\mathbf{y}$, as described in step 3 of Algorithm 1. $\mathbf{Y}^{m}$ has $n$ columns, corresponding to $n$ message types that reported the identifier (analogous to

\footnotetext{
${ }^{2}$ Like the state variable case, identifiers are chosen as variables reported at least $0.2 N$ times, where $N$ is total number of messages. We also require the variables have at least $0.02 \mathrm{~N}$ distinct values, and reported in at least 5 distinct messages types.
} 


\begin{tabular}{|l|l|l|}
\hline Feature & Rows & Columns \\
\hline Status ratio matrix $\mathbf{Y}^{s}$ & time window & state value \\
\hline Message count matrix $\mathbf{Y}^{m}$ & identifier & message type \\
\hline
\end{tabular}

Table 4: Semantics of rows and columns of features

"terms"). $\mathbf{Y}^{m}$ has $m$ rows, each of which corresponds to a message group (analogous to "document").

Although the message count matrix $\mathbf{Y}^{m}$ has completely different semantics from the state ratio matrix $\mathbf{Y}^{s}$, both can be analyzed using matrix-based anomaly detection tools (see Section 5). Table 4 summarizes the semantics of the rows and columns of each feature matrix.

\subsection{Implementing feature creation algorithms}

To improve efficiency of our feature generation algorithms in map-reduce, we tailored the implementation. The step of discovering state variables and/or identifiers (the first steps in Section 4.1 and 4.2) is a single mapreduce job that calculates the number of distinct values for all variables and determines which variables to include in further feature generation steps. The step of constructing features from variables is another map-reduce job with log parsing as the map stage and message grouping as the reduce stage. For the state ratio, we sort messages by time stamp, while for the message count vector, we sort by identifier values. Notice that the map stage (parsing step) only needs to output the required data rather than the entire text message, resulting in huge $\mathrm{I} / \mathrm{O}$ savings during the data shuffling and sorting before reduce. Feature creation time is negligible when compared to parsing time.

\section{Anomaly Detection}

We use anomaly detection methods to find unusual patterns in logs. In this way, we can automatically find $\log$ segments that are most likely to indicate problems. Given the feature matrices we construct, outlier detection methods can be applied to detect anomalies contained in the logs. We have investigated a variety of such methods and have found that Principal Component Analysis (PCA) $[5,16]$ combined with term-weighting techniques from information retrieval $[23,26]$ yields excellent anomaly detection results on both feature matrices, while requiring little parameter tuning.

PCA is a statistical method that captures patterns in high-dimensional data by automatically choosing a set of coordinates - the principal components - that reflect covariation among the original coordinates. We use PCA to separate out repeating patterns in feature vectors, thereby making abnormal message patterns easier to detect. PCA has runtime linear in the number of feature vectors; therefore, detection can scale to large logs.

Intuition behind PCA anomaly detection. (The mathchallenged may want to skip to the results in Section 6.) By construction, dimensions in our feature vectors are

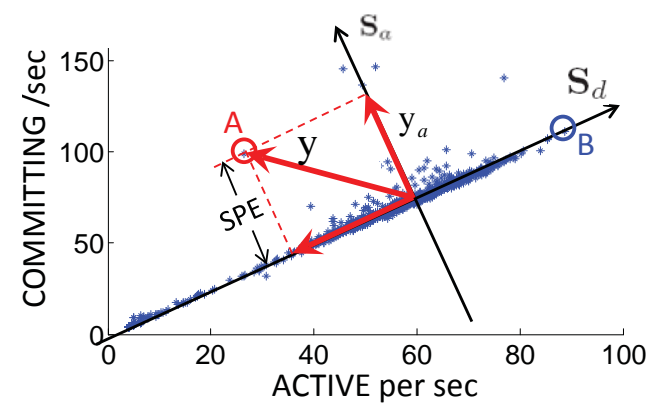

Figure 4: The intuition behind PCA detection with simplified data. We plot only two dimensions from the Darkstar state variable feature. It is easy to see high correlation between these two dimensions. PCA determines the dominant normal pattern, separates it out, and makes it easier to identify anomalies.

\begin{tabular}{|l|r|r|}
\hline Feature data sets & $n$ & $k$ \\
\hline Darkstar - message count & 18 & 3 \\
\hline Darkstar - state ratio & 6 & 1 \\
\hline HDFS - message count & 28 & 4 \\
\hline HDFS - state ratio & 202 & 2 \\
\hline
\end{tabular}

Table 5: Low effective dimensionality of feature data. $n=$ Dimensionality of feature vector $\mathbf{y} ; k=$ Dimensionality required to capture $95 \%$ of variance in the data. In all of our data, we have $k \ll n$, exhibiting low effective dimensionality.

highly correlated, due to the strong correlation among $\log$ messages within a group. We aim to identify abnormal vectors that deviate from such correlation patterns. Figure 4 illustrates a simplified example using two dimensions (number of ACTIVE and COMMITTING per second) from Darkstar state ratio vectors. We see most data points reside close to a straight line (a one-dimensional subspace). In this case, we say the data have low effective dimensionality. The axis $\mathbf{S}_{d}$ captures the strong correlations between the two dimensions. Intuitively, a data point far from the $\mathbf{S}_{d}$ (such as point A) shows unusual correlation, and thus represents an anomaly. In contrast, point B, although far from most other points, resides close to the $\mathbf{S}_{d}$, and is thus normal. In fact, both ACTIVE and COMMITTING are larger in this case, which simply indicates that the system is busier.

Indeed, we do observe low effective dimensionality in the feature matrices $\mathbf{Y}^{s}$ and $\mathbf{Y}^{m}$ in many systems. Table 5 shows $k$, the number of dimensions required to capture $95 \%$ of the variance in data ${ }^{3}$. Intuitively, in the case of the state ratio, when the system is in a stable state, the ratios among different state variable values are roughly constant. For the message count vector, as each dimension corresponds to a certain stage in the program and the stages are determined by the program logic, the messages in a group are correlated. The correlations among messages, determined by the normal program execution, result in highly correlated dimensions for both features.

\footnotetext{
${ }^{3}$ This is a common heuristic for determining $k$ in PCA detectors [15]; we use this number in all of our experiments.
} 
In summary, PCA captures dominant patterns in data to construct a (low) $k$-dimensional normal subspace $\mathbf{S}_{d}$ in the original $n$-dimensional space. The remaining $(n-k)$ dimensions form the abnormal subspace $\mathbf{S}_{a}$. By projecting the vector $\mathbf{y}$ on $\mathbf{S}_{a}$ (separating out its component on $\mathbf{S}_{d}$ ), it is much easier to identify abnormal vectors. This forms the basis for anomaly detection $[5,16]$.

Detecting anomalies. Intuitively, we use the "distance" from the end point of a vector $\mathbf{y}$ to the normal subspace $\mathbf{S}_{d}$ to determine whether $\mathbf{y}$ is abnormal. This can be formalized by computing the squared prediction error $\mathbf{S P E} \equiv\left\|\mathbf{y}_{a}\right\|^{2}$ (the squared length of vector $\mathbf{y}_{a}$ ), where $\mathbf{y}_{a}$ is the projection of $\mathbf{y}$ onto the abnormal subspace $\mathbf{S}_{a}$, and can be computed as $\mathbf{y}_{a}=\left(\mathbf{I}-\mathbf{P} \mathbf{P}^{T}\right) \mathbf{y}$, where $\mathbf{P}=\left[\mathbf{v}_{1}, \mathbf{v}_{2}, \ldots, \mathbf{v}_{k}\right]$, is formed by the first $k$ principal components chosen by PCA algorithm.

As Figure 4 shows, abnormal vectors are typically far away from the normal subspace $\mathbf{S}_{d}$. Thus, the "detection rule" is simple: we mark $\mathbf{y}$ is abnormal if

$$
\mathbf{S P E}=\left\|\mathbf{y}_{a}\right\|^{2}>Q_{\alpha},
$$

where $Q_{\alpha}$ denotes the threshold statistic for the SPE residual function at the $(1-\alpha)$ confidence level.

Automatically determine detection threshold. To compute $Q_{\alpha}$ we make use of the $Q$-statistic, a wellknown test statistic for the SPE residual function [13]. The computed threshold $Q_{\alpha}$ guarantees that the false alarm probability is no more than $\alpha$ under the assumption that data matrix $\mathbf{Y}$ has a multivariate Gaussian distribution. However, as pointed out by Jensen and Solomon [13], and as verified in our empirical work, the $Q$-statistic is robust even when the underlying distribution of the data differs substantially from Gaussian.

The choice of the confidence parameter $\alpha$ for anomaly detection has been studied in previous work [16], and we follow standard recommendations in choosing $\alpha=$ 0.001 in our experiments. We found that our detection results are not sensitive to this parameter choice.

Improving PCA detection results. Our message count vector is constructed in a way similar to the bag-of-words model, so it is natural to consider term weighting techniques from information retrieval. We applied Term Frequency / Inverse Document Frequency (TF-IDF), a wellestablished heuristic in information retrieval [23, 26], to pre-process the data. Instead of applying PCA directly to the feature matrix $\mathbf{Y}^{m}$ we replace each entry $y_{i, j}$ in $\mathbf{Y}^{m}$ with a weighted entry $w_{i, j} \equiv y_{i, j} \log \left(n / d f_{j}\right)$, where $d f_{j}$ is total number of message groups that contain the $j$-th message type. Intuitively, multiplying the original count with the IDF reduces the weight of common message types that appear in most groups, which are less likely to indicate problems. We found this step to be essential for improving detection accuracy.

\begin{tabular}{|r|r|r|r|}
\hline System & Total Log & Failed & Failed \% \\
\hline HDFS & $24,396,061$ & 29,636 & $0.121 \%$ \\
\hline Darkstar & $1,640,985$ & 35 & $0.002 \%$ \\
\hline
\end{tabular}

Table 6: Parsing accuracy. Parse fails on a message when we cannot find a message template that matches the message and extract message variables.

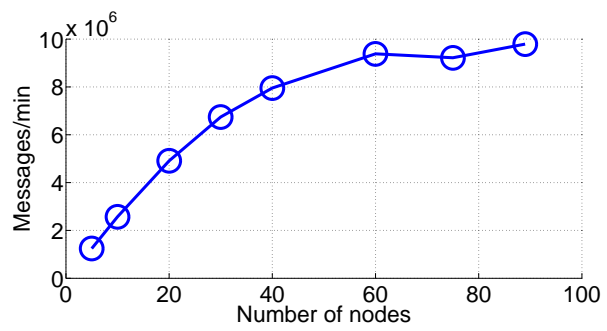

Figure 5: Scalability of $\log$ parsing with number of nodes used. The $\mathrm{x}$-axis is the number of nodes used, while the $\mathrm{y}$-axis is the number of messages processed per minute. All nodes are Amazon EC2 high-CPU medium instances. We used the HDFS data set (described in (Table 3) with over 24 million lines. We parsed raw textual logs and generated the message count vector feature (see Section 4.2). Each experiment was repeated 4 times and the reported data point is the mean.

TF-IDF does not apply to the state ratio feature. This is because the state ratio matrix is a dense matrix that is not amenable to an interpretation as a bag-of-words model. However, applying the PCA method directly to $\mathbf{Y}^{s}$ gives good results on the state ratio feature.

\section{Evaluation and Visualization}

We first show the accuracy and scalability achieved by our log parsing method (Section 6.1) and then discuss our experiences with the two real-world systems.

We began our experiments of problem detection with Darkstar, in which both features give simple yet insightful results (Section 6.2). Satisfied with these results, we applied our techniques to the much more complex HDFS logs. We also achieve high detection accuracy (Section 6.3). However, the results are less intuitive to system operators and developers, so we developed a decision tree visualization method, which summarizes the PCA detection results in a single, intuitive picture (Section 6.4) that is more operator friendly because the tree resembles the rule-based event processing systems operators use [10].

\subsection{Log parsing accuracy and scalability}

Accuracy. Table 6 shows that our log parsing method achieves over $99.8 \%$ accuracy on both systems. Specifically, our technique successfully handled rare messages types, even those that appeared only twice in over 24 million messages in HDFS. On the contrary, word-frequency based console log analysis tools, such as SLCT [32], do 
not recover either of the features we use in this paper. State variables are too common to be separated from constant strings by word frequency only. In addition, these tools ignore all rare messages, which are required to construct message count vectors.

There are only a few message types that our parser fails to handle. Almost all of these messages contain long string variables. These long strings may overwhelm the constant strings we are searching for, preventing reverse index search from finding the correct message template. However, these messages typically appear at the initialization or termination phase of a system (or a subsystem), when the state of the system is dumped to the console. Thus, we did not see any impact of missing these messages on our detection results.

We believe the accuracy of our approach to parsing is essential; only with an accurate parsing system can we extract state variables and identifiers- the basis for our feature construction-from textual logs. Thus, we consider the requirement of access to source code to be a small price to pay (especially given that many modules are open-source), given the high quality parsing results that our technique produces.

Scalability. We evaluated the scalability of our log parsing approach with a varying number of EC2 nodes. Figure 5 shows the result: Our log parsing and feature extraction algorithms scale almost linearly with up to about 50 nodes. Even though we parsed all messages generated by 200 HDFS nodes (with aggressive logging) over 48 hours, log parsing only takes less than 3 minutes with 50 nodes, or less than 10 minutes with 10 nodes. When we use more than 60 nodes, the overhead of index dissemination and job scheduling dominate running time.

\subsection{Darkstar experiment results}

As mentioned in Section 2.3, we observed high performance (i.e., client side response time) variability when deploying the Darkstar server on a cloud-computing environment such as EC2 during performance disturbances, especially for CPU contention. We wanted to see if we could understand the reason for this high performance variability solely from console logs. Indeed, we were unfamiliar with Darkstar, so our setting was realistic as the operator often knows little about system internals.

In the experiment, we deployed an unmodified Darkstar 0.95 distribution on a single node (because the Darkstar version we use supports only one node). Darkstar does not log much by default, so we turned on the debuglevel logging. We deployed a simple game, DarkMud, provided by the Darkstar team, and created a workload generator that emulated 60 user clients in the DarkMud virtual world performing random operations such as flipping switches, picking up and dropping items. The client emulator recorded the latency of each operation. We ran the experiment for 4800 seconds and injected a per- formance disturbance by capping the CPU available to Darkstar to $50 \%$ of the normal level during time 1400 to 1800 .

Detection by state ratio vectors. The only state variable chosen by our feature generation algorithm is state, which is reported in 456, 996 messages (about 28\% of all $\log$ messages in our data set). It has 8 distinct values, including PREPARING, ACTIVE, COMMITTING, ABORTING and so on, so our state ratio matrix $\mathbf{Y}^{s}$ has 8 columns (dimensions). The time window (automatically determined according to Section 4.1) is 3 seconds; we restricted the choice to whole seconds.

Figures 6 (a) and (b) show the results between time 1000 and 2500, where plot (a) displays the average latency reported by the client emulator, which acts as a ground truth for evaluating our method, and plot (b) displays the PCA anomaly detection results on the state ratio matrix $\mathbf{Y}^{s}$. We see that anomalies detected by our method during the time interval $(1400,1800)$ match the high client-side latency very well; i.e., the anomalies detected in the state ratio matrix correlate very well with the increases in client latency. Comparing the abnormal vectors to the normal vectors, we see that the ratio between number of ABORTING to COMMITTING increases from about 1:2000 to about 1:2, indicating that a disproportionate number of $A B O R T I N G$ transactions are related to the poor client latency.

Generally, the abnormal state ratio may be the cause, symptom, or consequence of the performance degradation. In the Darkstar case, the ratio reflects the cause of the problem: when the system performance gets worse, Darkstar does not adjust transaction timeout accordingly, causing many normal transactions to be aborted and restarted, resulting in further load increase to the system.

Notice that a traditional grep-based method does not help in this case for two reasons: 1) As a normal user of Darkstar-without having knowledge about its internals - the transaction states are obscure implementation details. Thus, it is difficult for a user to choose the correct ones from many variables to search for. In contrast, we systematically discover and analyze all state variables. 2) ABORTING happens even during normal operation times, due to the optimistic concurrency model used in Darkstar, where aborting is used to handle access conflicts. It is not a single ABORTING message, but the ratio of $A B O R T I N G$ to other values of the state variable that captures the problem.

Detection by message count vectors. From Darkstar logs, Algorithm 1 automatically chooses two identifier variables, the transaction id and the asynchronous channel id. Figure 6(c) shows detection results on the message count vector constructed from the transaction id variable. There are 68,029 transaction ids reported in 18 different message types. Thus, the dimension of matrix 


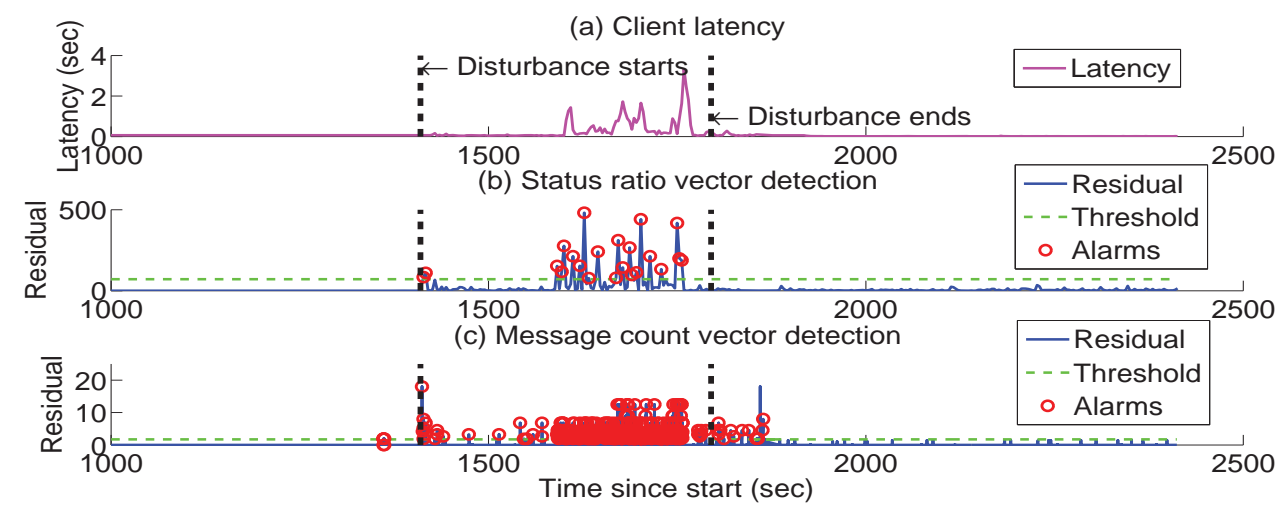

Figure 6: Darkstar detection results. (a) shows that the disturbance injection caused a huge increase in client response time. (b) shows PCA anomaly detection results on the state ratio vector created from message variable state. The dashed line shows the threshold $Q_{\alpha}$. The solid line with spikes is the SPE calculated according to Eq. (1). The circles denote the anomalous vectors detected by our method, whose SPE values exceed threshold $Q_{\alpha}$. (c) shows detection results with the message count vector. The SPE value of each vector (the solid line) is plotted at the time when the last message of the group occurs.

$\mathbf{Y}^{m}$ is $68,029 \times 18$. By construction, each message count vector represents a set of operations (message types) occurring when executing a transaction. PCA identifies the normal vectors corresponding to a common set of operations (simplified for presentation): \{create, join txn, commit, prepareAndCommit\}. Abnormal transactions can deviate from this set by missing a few message types, or having rare message types such as abort txn instead of commit and join txn. We detected 504 of these as abnormal. To validate our result, we augmented each feature vector using the timestamp of the last message in that group, and we found that almost all abnormal transactions occur when the disturbance is injected. We see that the anomalies continue to appear (with a smaller frequency) for a short time period after the disturbance stopped due to the queueing effect as the system recovered from the disturbance. Notice that the state ratio vector method did not mark the recovery period as abnormal, demonstrating that the message count vector method was more sensitive because it modeled individual operations while state ratio vector method captured only aggregate behavior.

There were no anomalies on the channel id variable during the entire experiment, suggesting that the channel id variable is not related to this performance anomaly.

This result is consistent with the state ratio vector detection result. In console logs, it is common that there are several different pieces of information that describe the same system behavior. This commonality suggests an important direction for future research: to exploit multisource learning algorithms, which combine multiple detection results to further improve accuracy.

\subsection{Hadoop experiment results}

Compared to Darkstar, HDFS is larger scale and the logic is much more complex. In this experiment, we show that we can automatically discover many abnormal behaviors in HDFS. We generated the HDFS logs by setting up a Hadoop cluster on 203 EC2 nodes and running sample Hadoop map-reduce jobs for 48 hours, generating and processing over $200 \mathrm{~TB}$ of random data. We collected over 24 million lines of logs from HDFS.

Detection on message count vector. From HDFS logs, Algorithm 1 automatically chooses one identifier variable, the blockid, which is reported in 11, 197,954 messages (about $50 \%$ of all messages) in 29 message types. Also, there are 575, 139 distinct blockids reported in the log, so the message count matrix $\mathbf{Y}^{m}$ has a dimension of $575,139 \times 29$. The PCA detector gives very good separation between normal and abnormal row vectors in the matrix: Using an automatically determined threshold $\left(Q_{\alpha}\right.$ in Eq. (1) in Section 5), it can successfully detect abnormal vectors corresponding to blocks that went through abnormal execution paths.

To further validate our results, we manually labeled each distinct message vector, not only marking them as normal or abnormal, but also determining the type of problems for each vector. The labeling was done by carefully studying HDFS code and by consulting with local Hadoop experts. We show in the next section that the decision tree visualization helps both ourselves and Hadoop developers to understand our results. We emphasize that this labeling step is done only to validate our method-it is not a required step when using our technique. Labeling half a million vectors is possible because many of the vectors are exactly the same. In fact, there are only 680 distinct vectors, confirming our intuition that most blocks go through a common execution path.

Table 7 shows the manual labels and detection results. We see that the PCA detector can detect a large fraction of anomalies in the data, and significant improvement can be achieved when we preprocess data with TF-IDF, confirming our expectations from Section 5.

Throughout the experiment, we experienced no catas- 
trophic failures; thus, most problems listed in Table 7 only affect performance.

The first anomaly in Table 7 uncovered a bug that has been hidden in HDFS for a long time. In a certain (relatively rare) code path, when a block is deleted (due to temporary over-replication), the record on the namenode is not updated until the next write to the block, causing the file system to believe in a replica that no longer exists, which causes subsequent block deletion to fail. Hadoop developers have recently confirmed this bug. This anomaly is hard to find because there is no single error message indicating the problem. However, we discover it because we analyze abnormal execution paths.

We also notice that we do not have the problem that causes confusion in traditional grep based log analysis. In HDFS datanode logs, we see many messages like \#:Got Exception while serving \# to \#:\#. According to Apache issue tracking (jira) HADOOP-3678, this is a normal behavior of HDFS: the HDFS data node generates the exception when a HDFS client does not finish reading an entire block before it stops. These exception messages have confused many users, as indicated by multiple discussion threads on the Hadoop user mailing list. While traditional keyword matching (e.g., searching for words like Exception or Error) would have flagged these as errors, our message count method successfully avoids this false positive because this happens too many times to be abnormal.

Our algorithm does report some false positives, which are inevitable in any unsupervised learning algorithm. For example, the second false positive in Table 7 occurs because a few blocks are replicated 10 times instead of 3 times for the majority of blocks. These message groups look suspicious, but Hadoop experts told us that these are normal situations when the map-reduce system is distributing job configuration files to all the nodes. It is indeed a rare situation compared to the data accesses, but is normal by the system design. Eliminating this type of "rare but normal" false positive requires domain expert knowledge. As a future direction, we are investigating semi-supervised learning techniques that can take operator feedback and further improve our results.

Detection on state ratio vectors. The only state variable chosen in HDFS logs by our feature generation algorithm is the node name. Node name might not sound like a state variable, but as the set of nodes (203 total) are relatively fixed in HDFS, and their names meet the criterion of state variable described in Section 4.1. Thus, the state ratio vector feature reduces to per node activity count, a feature well-studied in existing work [12, 17]. As in this previous work, we are able to detect transient workload imbalance, as well as node reboot events. However, our approach is less ad-hoc because the state ratio feature is chosen automatically based on information in the console

\begin{tabular}{|r|l|r|r|r|}
\hline$\#$ & Anomaly Description & Actual & Raw & TF-IDF \\
\hline 1 & $\begin{array}{l}\text { Namenode not updated after } \\
\text { deleting block }\end{array}$ & 4297 & 475 & 4297 \\
\hline 2 & Write exception client give up & 3225 & 3225 & 3225 \\
\hline 3 & Write failed at beginning & 2950 & 2950 & 2950 \\
\hline 4 & Replica immediately deleted & 2809 & 2803 & 2788 \\
\hline 5 & $\begin{array}{l}\text { Received block that does not } \\
\text { belong to any file }\end{array}$ & 1240 & 20 & 1228 \\
\hline 6 & Redundant addStoredBlock & 953 & 33 & 953 \\
\hline 7 & $\begin{array}{l}\text { Delete a block that no longer } \\
\text { exists on data node }\end{array}$ & 724 & 18 & 650 \\
\hline 8 & Empty packet for block & 476 & 476 & 476 \\
\hline 9 & Receive block exception & 89 & 89 & 89 \\
\hline 10 & Replication Monitor timedout & 45 & 37 & 45 \\
\hline 11 & Other anomalies & 108 & 91 & 107 \\
\hline & Total & $\mathbf{1 6 9 1 6}$ & $\mathbf{1 0 2 1 7}$ & $\mathbf{1 6 8 0 8}$ \\
\hline
\end{tabular}

\begin{tabular}{|r|l|r|r|}
\hline$\#$ & False Positive Description & Raw & TF-IDF \\
\hline 1 & Normal background migration & 1399 & 1397 \\
\hline 2 & Multiple replica (for task / job desc files) & 372 & 349 \\
\hline 3 & Unknown Reason & 26 & 0 \\
\hline & Total & $\mathbf{1 7 9 7}$ & $\mathbf{1 7 4 6}$ \\
\hline
\end{tabular}

Table 7: Detected anomalies and false positives using PCA on Hadoop message count vector feature. Actual is the number of anomalies labeled manually. Raw is PCA detection result on raw data, TF-IDF is detection result on data preprocessed with TF-IDF and normalized by vector length (Section 5).

$\log$, instead of manually specified.

\subsection{Visualizing detection results with decision trees}

From the point of view of an operator, the transformation underlying PCA is a black box algorithm: it provides no intuitive explanation of the detection results and cannot be interrogated. Human operators need to manually examine anomalies to understand the root cause, and PCA itself provides little help in this regard. In this section, we show how to augment PCA-based detection with decision trees to make the results more easily understandable and actionable by operators. The decision tree result resembles the (manually written) rules used in many system-event-processing programs [10], so it is easier for non-machine learning experts. This technique is especially useful for features with many dimensions, such as the message count vector feature in HDFS.

Decision trees have been widely used for classification. Because decision tree construction works in the original coordinates of the input data, its classification decisions tend to be easy to visualize and understand [34]. Constructing a decision tree requires a training set with class labels. We use the automatically generated PCA detection results (normal vs. abnormal) as class labels, in contrast to the normal use of decision trees. Our decision tree is constructed to explain the underlying logic of the detection algorithm, rather than the nature of the dataset.

Figure 7 is the decision tree generated using RapidMiner [21] from the anomaly detection results of the HDFS log. It clearly shows the most important mes- 


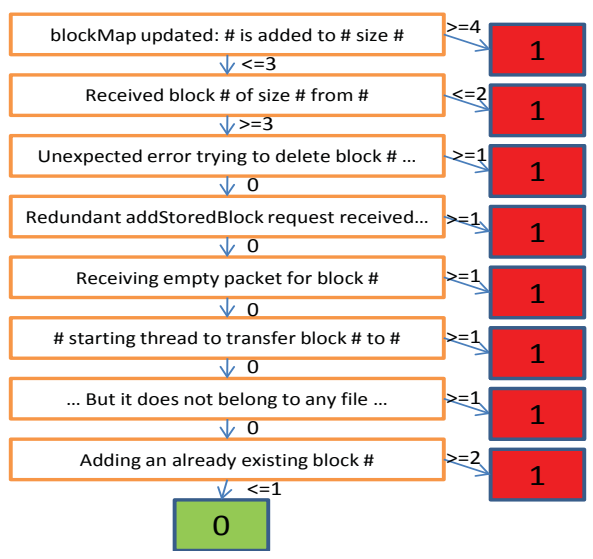

Figure 7: The decision tree visualization. Each node is the message type string (\# is the place holder for variables). The number on the edge is the threshold of message count, generated by the decision tree algorithm. Small boxes contain the labels from PCA, with a red 1 for abnormal and a green 0 for normal.

sage types. For example, the first level shows that if blockMap (the data structure that keeps block locations) is updated more than 3 times, it is abnormal. This indicates the over-replication problem (Anomaly 4 or False Positive 1 in Table 7). The second level shows that if a block is received 2 times or less, it is abnormal; this indicates under-replication or block-write failure (Anomaly 2 and 3 in Table 7). Level 3 of the decision tree is related to the bug we discussed in Section 6.3.

In summary, the visualization of results with decision trees helps operators and developers notice types of abnormal behaviors instead of individual abnormal events, which can greatly improve the efficiency of finding root causes and preventing future alarms.

\section{Discussion}

Should we completely replace console logs with structured tracing? There are various such efforts [8, 29]. Progress has been slow, however, mainly because there is no standard for structured tracing embraced by all open source developers. It is also technically difficult to design a global "schema" to accommodate all information contained in console logs in a structured format ${ }^{4}$. Even if such a standard existed, manually porting all legacy codes to the schema would be expensive. Automatic porting of legacy logging code to structured logging would be no simpler than our log parsing. Our feature creation and anomaly detection algorithm can be used withoutlog parsing in systems with structured traces only, and we described a successful example in Section 2.2

Improving console logs. We have discovered some bad

\footnotetext{
${ }^{4}$ syslog is not structured because it uses the free text field heavily.
}

logging practices that significantly reduced the usefulness of the console log. Some of them are easy to fix. For example, Facebook's Cassandra storage system traces all operations of nodes sending messages to each other, but it does not write the sequence number or ID of messages logged. This renders the log almost useless if multiple threads on a single machine are sending messages concurrently. However, just by adding the message ID, our message count method readily applies and would help detect node communication problems.

Another bad logging practice, which has been discovered in prior work, is the poor estimate of event severity. Many "FATAL" or "ERROR" events are not as bad as the developer thinks $[14,22]$. This mistake is because each developer judges the severity only in the context of his own module instead of in the context of the entire system. As we show in the Hadoop read exception example, our tool, based on the frequency of the events, can provide developers with insight into the real severity of individual events and thus improve quality of future logging.

Challenges in log parsing. Since we rely on static source code analysis to extract structure from the logs, our method may fail in some cases and fall back on identifying a large chunk of a log message as an unparsed string. For example, if programmers use very general types such as object in Java (very rare in practice), our type resolution step fails because there are too many possibilities. We guard against this by limiting the number of descendants of a class to 100, which is large enough to accommodate all logs we studied but small enough to filter out genuine JDK, AWT and Swing classes with many subclasses (such as object). Features such as generics and mix-ins in modern OO languages provide the mechanisms usually needed to avoid having to declare an object in a very general class. In addition, some log messages are undecorated, emitting only a variable of some primitive type without any constant label. These messages are usually leftovers from the debugging phase, and we simply ignore these messages.

\section{Related Work}

Most existing work treats the entire log as a single sequence of repeating message types and mines it with time series analysis methods. Hellerstein et al. developed a novel method to mine important patterns such as message burst, periodicity and dependencies from SNMP data in an enterprise network $[12,18]$. Yamanishi et al. modeled syslog sequences as a mixture of Hidden Markov Models (HMM), in order to find messages that are likely to be related to critical failures [35]. Lim et al. analyzed a large-scale enterprise telephony system log with multiple heuristic filters to find messages related to actual failures [17]. Treating a log as a single time series, however, does not perform well in large-scale clusters with 
multiple independent processes that generate interleaved logs. The model becomes overly complex and parameters are hard to tune with interleaved logs [35]. Our analysis is based on message groups rather than a time series of individual messages. The grouping approach makes it possible to obtain useful results with simple, efficient algorithms such as PCA.

A crucial but questionable assumption in previous work is that message types can be detected accurately. Some projects $[12,18]$ use manual type labels from SNMP data, which are not generally available in console logs. Many other projects use simple heuristics-such as removing all numeric values and strings that resemble IP addresses-to detect message types $[17,35]$. These heuristics are not sufficiently general. If the heuristics fail to capture some relevant variables, the resulting message types can be in the tens of thousands [17]. SLCT [32], Loghound [33], Sisyphus [27], and [7] use more advanced clustering and association rules, as well as scoring methods from information retrieval to extract message templates for log parsing. IPLoM [19] used a series of heuristics to capture the differences of similar log messages to determine message types. Although these methods can successfully detect recurring patterns, they do so by considering textual properties of logs. In contrast, our approach extracts information about program objects from log messages, and our detection is based on event traces related to those objects, rather than on textual properties. Indeed, our message count vector feature is more similar to path-based problem detection approaches such as Pinpoint [3, 8], as we pointed out in Section 4.2.

Software development involves other textual information than console logs. By making use of source code, Tan et al. proposed a novel approach to detect inconsistencies between textual comments and the program logic [31]. Our idea is similar in that we can make textual information designed for human also machineunderstandable by using highly structured source code. However, there are unique challenges in console log analysis, because we must analyze runtime information in addition to source code.

\section{Conclusions and Future Work}

We propose a general approach to problem detection via the analysis of console $\operatorname{logs}$, the built-in monitoring information in most software systems. Using source code as a reference to understand the structure of console logs, we are able to parse logs accurately. The accuracy in log parsing allows us to extract the identifiers and state variables, which are widely found in logs yet are usually ignored due to difficulties in log parsing. Using console logs, we are able to construct powerful features that were previously exploited only in structured traces. These features reveal accurate information on system execution; thus, efficient algorithms such as PCA yield promising anomaly detection results. In addition, we summarize detection results with decision tree visualization, which helps operators/integrators/developers to quickly understand the detection result.

Our work has opened up many new opportunities for turning built-in console logs into a powerful monitoring system for problem detection, and suggests a variety of future directions that can be explored, including: 1) extracting log templates from program binaries instead of source code, which not only makes our approach work on non-open-source modules but also brings much operational convenience; 2) designing other features to fully utilize the rich information in console logs; 3 ) developing online detection algorithms instead of current postmortem analysis; and 4) investigating methods to correlate logs from multiple related applications and detect more complex failure cases.

\section{Acknowledgements}

The authors would like to thank Bill Bolosky, Richard Draves, Jon Stearley, Byung-Gon Chun, Jaideep Chandrashekar, Petros Maniatis, Peter Vosshall, Deborah Weisser, Kimberly Keeton and Kristal Sauer for their great suggestions on the early draft of the paper. Our thanks also go to the anonymous SOSP reviewers and especially our shepherd Jeff Chase for the feedbacks.

This research is supported in part by gifts from Sun Microsystems, Google, Microsoft, Amazon Web Services, Cisco Systems, Facebook, Hewlett-Packard, Network Appliance, and VMWare, and by matching funds from the University of California Industry/University Cooperative Research Program (UC Discovery) grant COM07-10240.

\section{References}

[1] A. W. Appel. Modern Compiler Implementation in Java. Cambridge University Press, second edition, 2002.

[2] D. Borthakur. The hadoop distributed file system: Architecture and design. Hadoop Project Website, 2007.

[3] M. Y. Chen and et al. Path-based failure and evolution management. In Proc. NSDI'04, pages 23-23, San Francisco, California, 2004. USENIX.

[4] M. H. DeGroot and M. J. Schervish. Probability and Statistics. Addison-Wesley, 3rd edition, 2002.

[5] R. Dunia and S. J. Qin. Multi-dimensional fault diagnosis using a subspace approach. In Proc. ACC, 1997.

[6] R. Feldman and J. Sanger. The Text Mining Handbook: Advanced Approaches in Analyzing Unstructured Data. Cambridge Univ. Press, 122006.

[7] K. Fisher, D. Walker, K. Q. Zhu, and P. White. From dirt to shovels: fully automatic tool generation from ad hoc data. In Proceedings of ACM POPL '08, pages 421-434, 2008.

[8] R. Fonseca and et al. Xtrace: A pervasive network tracing framework. In In Proc. NSDI, 2007.

[9] C. Gulcu. Short introduction to $\log 4 j$, March 2002. http://logging.apache.org/log $4 \mathrm{j}$. 
[10] S. E. Hansen and E. T. Atkins. Automated system monitoring and notification with Swatch. In Proc. USENIX LISA '93, pages 145-152, 1993.

[11] E. Hatcher and O. Gospodnetic. Lucene in Action. Manning Publications Co., Greenwich, CT, 2004.

[12] J. Hellerstein, S. Ma, and C. Perng. Discovering actionable patterns in event data. IBM Sys. Jour, 41(3), 2002.

[13] J. E. Jackson and G. S. Mudholkar. Control procedures for residuals associated with principal component analysis. Technometrics, 21(3):341-349, 1979.

[14] W. Jiang and et al. Understanding customer problem troubleshooting from storage system logs. In Proceedings of USENIX FAST'09, 2009.

[15] I. Jolliffe. Principal Component Analysis. Springer, 2002.

[16] A. Lakhina, M. Crovella, and C. Diot. Diagnosing network-wide traffic anomalies. In Proc. ACM SIGCOMM, 2004.

[17] C. Lim, N. Singh, and S. Yajnik. A log mining approach to failure analysis of enterprise telephony systems. In Proc. DSN, June 2008.

[18] S. Ma and J. L. Hellerstein. Mining partially periodic event patterns with unknown periods. In Proc. IEEE ICDE, Washington, DC, 2001.

[19] A. A. Makanju, A. N. Zincir-Heywood, and E. E. Milios. Clustering event logs using iterative partitioning. In Proceedings of KDD '09, 2009.

[20] C. Manning, P. Ragahavan, and et al. Introduction to Information Retrieval. Cambridge University Press, 2008.

[21] I. Mierswa, M. Wurst, R. Klinkenberg, M. Scholz, and T. Euler. Yale: Rapid prototyping for complex data mining tasks. In Proc. ACM KDD, New York, NY, 2006.

[22] A. Oliner and J. Stearley. What supercomputers say: A study of five system logs. In Proc. IEEE DSN, Washington, DC, 2007.

[23] K. Papineni. Why inverse document frequency? In Proc. NAACL '01:, pages 1-8, Morristown, NJ, 2001. Asso. for Comp. Linguistics.

[24] J. E. Prewett. Analyzing cluster log files using logsurfer. In Proc. Annual Conf. on Linux Clusters, 2003.

[25] T. Sager, A. Bernstein, M. Pinzger, and C. Kiefer. Detecting similar java classes using tree algorithms. In Proc. ACM MSR '06, pages 65-71, 2006.

[26] G. Salton and C. Buckley. Term weighting approaches in automatic text retrieval. Technical report, Cornell, Ithaca, NY, USA, 1987.

[27] J. Stearley. Towards informatic analysis of syslogs. In Proc. IEEE CLUSTER, Washington, DC, 2004.

[28] Sun. Project darkstar. www.projectdarkstar.com, 2008.

[29] Sun. Solaris Dynamic Tracing Guide, 2008.

[30] J. Tan and et al. SALSA: Analyzing logs as StAte machines. In Proc. of WASL '08, 2008.

[31] L. Tan, D. Yuan, G. Krishna, and Y. Zhou. /*icomment: bugs or bad comments?*/. In Proc. ACM SOSP '07, New York, NY, 2007. ACM.

[32] R. Vaarandi. A data clustering algorithm for mining patterns from event logs. Proc. IPOM, 2003.

[33] R. Vaarandi. A breadth-first algorithm for mining frequent patterns from event logs. In INTELLCOMM, volume 3283, pages 293-308. Springer, 2004.

[34] I. H. Witten and E. Frank. Data Mining: Practical Machine Learning Tools and Techniques with Java Implementations. Morgan Kaufmann, 2000.

[35] K. Yamanishi and Y. Maruyama. Dynamic syslog mining for network failure monitoring. In Proc. ACM KDD, New York, NY, 2005.

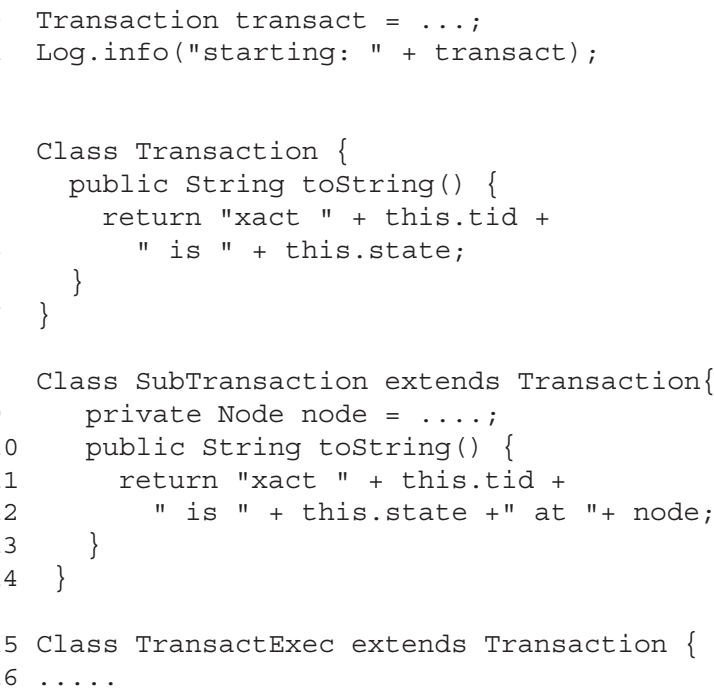

Figure 8: Example source code segments. Notice that the logger call in line 1 may generate different log messages at runtime due to the class hierarchies.

\section{A Appendix: Extracting message tem- plates from source code}

We illustrate the details of our source code analysis techniques for message template extraction with a running example in Java, though the general idea applies to other object-oriented languages as well.

Line 1 of Figure 8 is a simple logger call. However, as we discussed in Section 3, it might generate different kinds of messages such as

starting: xact 325 is COMMITTING

starting: xact 346 is ABORTING at $\mathrm{n} 1: 8080$ (1)

This is because the variable transact is a complex data type with multiple tostring () definitions (Line 2-15). Our goal is to discover all possible message templates that Line 1 can generate, so we need to resolve the type hierarchy information of transact.

Figure 9 illustrates the major steps of our approach. All analysis is done on the abstract syntax tree (AST) [1] generated by the Eclipse IDE. Our analysis uses three data structures created from the AST: a list of partial message templates, a table of templates representing tostring() methods for all declared types (the "toString Table"), and a Class Hierarchy table. Although logically the data structures are independent of each other, our implementation builds them using a single pass over the AST.

Partial message template extraction. We first look for all method invocations on objects of the logger class. This gives us the list of all log messages that could possibly be generated, whether they actually appear in the $\log$ or not. Common logger class libraries such as $\log 4 j$ based loggers [9] can be automatically detected by examining the library the software uses. Analyzing the pa- 


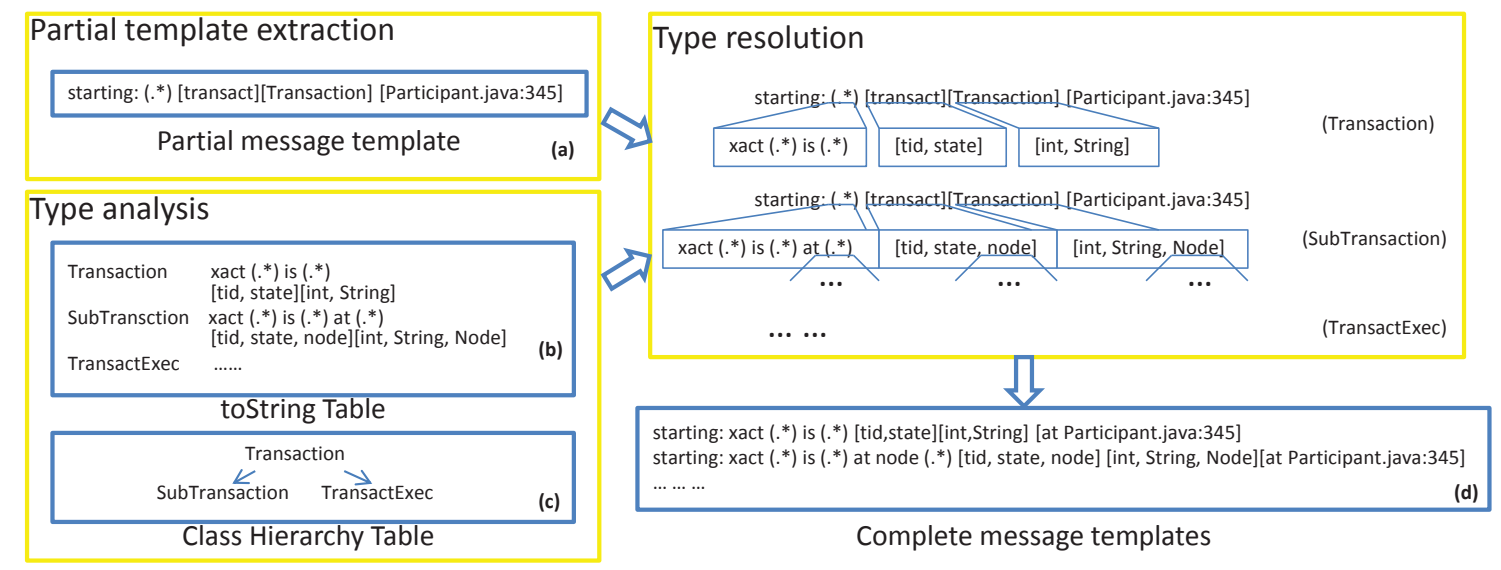

Figure 9: Constructing message templates from source code analysis.

rameters of the logger call in Line 1 of Figure 8 gives the partial message template shown in Figure 9 (a). We also record the names and types of message variables interpolated into the log message (such as transact in Line 1 of Figure 8), which are crucial for the final type resolution, the filename and line number of the logger call.

Type analysis. We next determine how each message variable will be rendered as a string in the logger call. For example, because transact is of type Transaction, we can determine how it would appear in a log message by looking at the tostring () method of the Transaction class. We traverse the AST to build a toString Table containing the tostring () definitions and toString templates of all classes. Figure 9 (b) shows the toString templates extracted from Lines $2-16$ in Figure 8 .

Due to the importance of class hierarchy information, we do a third traversal on AST to build the Class Hierarchy table. Box (c) in Figure 9 shows an example.

Type resolution. Finally, for each partial message template containing non-primitive variables (i.e., member of a nonprimitive class), we look up the class's toString method and corresponding toString templates in the toString Table, and substitute the templates found into the partial message template. For example, for the logger call in Line 1 of Figure 8 that references the transact object, we lookup the toString method of its class (Transaction). If the tostring() method is not explicitly defined in Transaction class, we use the Class Hierarchy Table, built from the AST, to attempt to resolve tostring() in the object's superclasses. We do this recursively until either a tostring () method is found or we reach the root of the class hierarchy (in Java, the java.lang.object class), in which case we give up and treat the template as an unparsed string $(. *)$.

The sub-classing problem is also handled in this step. We find all descendants of a declared class. If there is a tostring( $)$ method defined in any subclasses, we generate a message template as if the subclass is used instead of the declared class. For example, because subTransaction is a sub-class of Transaction, we generate a second message template capturing the case when transact is actually an instance of SubTransaction. We do this for every subclass of Transaction known at compile time.

Lastly, note that type resolution is recursive. For example, if an object has class SubTransaction, we examine the toString method of SubTransaction (line 8 of Figure 8) and we find that it interpolates a variable node of nonprimitive type (line 11 ). We recurse and substitute in the toString template of Node. We do this until the type of every variable becomes a primitive type or unparsed strings. We also limit the maximum depth of recursions to deal with recursive type definitions.

Because we are using static analysis techniques to predict what the log output will look like at runtime, it is impossible to correctly handle all cases. Examples of such cases includes loops and recursive calls. We make our technique robust by allowing it to fall back to unparsed string $(. *)$ in such cases. In the real systems we studied, these hard cases rarely occur in log printing and rarely cause problems in practice. There are some language-specific idioms such as Arrays.deeoTostring (array) (array dumping) in Java, which has an implicit built-in format of using comma to separate array elements. Our parser recognize these idioms and handle them as special cases. 